\title{
Effects of extensible modelling on composite riser mechanical responses
}

\author{
Hossam Ragheb ${ }^{\mathrm{a}, 1}$, Adam Sobey $\mathrm{a}^{\mathrm{a}, \mathrm{b}}$ \\ ${ }^{a}$ Fluid Structure Interaction Group, University of Southampton, Bolderwood Innovation Campus, Southampton, UK, SO16 7QF \\ ${ }^{b}$ Marine and Maritime Group, Data-centric Engineering Programme, The Alan Turing Institute, The British Library, London, UK, NW1 2DB
}

\begin{abstract}
The change from steel risers to composites comes with uncertainties that led to large safety factors. One area of uncertainty is the predicted response and stresses derived from commercial packages that are based on formulations that assume in-extensible riser. However, composite pipes exhibit a lower axial stiffness and therefore the velocity of the axial waves will change with a corresponding change in dynamic response. To determine the effect of this assumption, this paper assesses the effect of extensibility on the time-domain response. It is found that the in-extensible model predicts 3 times the number of high frequency tension cycles in the $20 \mathrm{kN}$ tension range. To determine the impact of this change on the stress, the accuracy of available composite pipe models is benchmarked using shell, continuumshell and solid elements. The quadratic and continuum-shell elements provide a maximum percentage difference of $4 \%$ compared to solid elements but the continuum-shell is selected as it has a lower computational cost. The response from the extensible and in-extensible models are input into the pipe model, they provide similar Tsai-Wu failure factors, alleviating concerns when modelling the strength. However, the change in dynamics remains a concern for other applications such as machine-learning or digital-twins.
\end{abstract}

Keywords: Composite risers, Cable dynamics, Time domain, Benchmarking

\section{The importance of composite risers}

Production risers are vital subsea components for hydrocarbon production. However, the design process is complex as they are long, slender and exposed to dynamic multi-axial loading conditions. In addition, they are subjected to large temperature and pressure differentials through the thickness which are caused by the transfer of hot fluids between the surface platforms and the subsea systems, sometimes in parts of the ocean where the ambient temperature is below $0^{\circ} \mathrm{C}$. Where temperature and pressure differentials leads to the seabed sections of a pipeline or SCR walking, which can lead to failure [1, 2, 3]. Steel is the most common material used in subsea applications. However,

\footnotetext{
${ }^{1}$ Corresponding author.

Email address:har1g15@soton.ac.uk 
at large depths the technical challenges increase due to the large top-tensions, installation difficulties and long-term integrity issues related to corrosion. As the offshore industry expands their operations to greater depths, potentially beyond 4000 meters, these problems will only get worse. Gradually replacing steel with composite materials could reduce the operational and capital expenditure due to their higher specific strength to weight ratios, lack of corrosion and excellent vibrational damping properties [4, 5, 6]. However, these benefits do not come without challenges, for example, the lighter weight of the composite could lead to higher compression loads [7] that requires ballast weights or internal metallic liners and high initial costs that are dependent on the manufacturing and qualification processes. This is in part because of the lack of full-scale in-situ field data due to the short track record in the offshore environment, in addition to the complexity and lack of accuracy of the failure prediction tools [8]. A possible reduction in the qualification costs can be provided by sufficiently accurate and rapid numerical models developed to predict full-scale in-situ behaviour and failure during operation. However there is little published research on the suitability of the current riser numerical models to capture the composite riser's non-linear dynamic response at the global riser and pipe levels.

This paper therefore aims to determine the effect that extensibility has on global riser and pipe levels, in an effort to improve confidence in modelling composite risers. First, the effect of the extensible and in-extensible beam formulations on the composite riser global response is investigated, to determine if the change in axial stiffness in composite pipes changes the behaviour and requires a different formulation than is currently used for flexible risers. Then, the suitability of different element types for predicting the composite pipe response is investigated, due to the lack of available benchmarks in the open literature. The investigation shows which of these different methods can accurately, and efficiently, capture the stresses within the laminate. Finally these two elements are combined to show how the choice of extensible or in-extensible methods affects the stress predictions at the laminate scale.

\section{Catenary riser numerical model}

\subsection{Review of methods for modelling the global response}

Riser design is performed in two stages for computational efficiency. First the global response of the entire riser is modelled under the influence of environmental and vessel loads. The dynamic excitations are captured within the vessel's top excitation. This dynamic time domain analysis is required to identify the critical riser responses and sections for ultimate and fatigue limits states. The second step is to build a detailed model of the critical cross-section. This analysis is performed to predict the stresses and strains in the laminate to optimise the layup. Outputs from the global analysis, the tensions and rotations at both ends of the critical sections are applied as boundary conditions to the cross-section model. 
The catenary equation is the simplest approach for modelling the global response of catenary shaped risers. However, the closed-form formulation limits its application. Solving dynamic riser simulations requires the solution of the full set of partial differential equations with time varying boundary conditions and loads and can only be solved with a suitable numerical method such as finite elements or finite differencing. The riser's global dynamics can be formulated using two main approaches: extensible and in-extensible beams. Cables exhibit two main vibrating modes, 'elastic', where the axial waves propagate with a velocity proportional to the axial stiffness, and 'transverse', where they propagate as a function of the beam flexural stiffness. Flexible steel risers exhibit high axial stiffnesses due to their multi-layer cross-sectional design which are often five orders of magnitude higher than their flexural stiffness, due to their multi-layer cross-sectional design, while maintaining favourable low bending stiffnesses. Therefore, it is reasonable to approximate these flexible pipes response using an in-extensible beam model. Approaches that are specifically developed for flexible steel risers enforce these conditions to avoid the singularity that arises in the numerically ill-conditioned system matrix [9] and this is common in most design software. However, composite risers exhibit a lower axial stiffness that is likely to be only three orders of magnitude higher than the flexural stiffness. At these lower ratios the elastic waves could be excited and it may be necessary to model the composite riser as an extensible cable.

The dynamic equilibrium system that governs the riser response consists of a number of non-linear coupled hyperbolic partial differential equations with boundary value constraints. The full-scale response of the riser can be adequately modelled using Bernoulli beam formulation as the length to thickness ratio is larger than the limit below which the Timoshenko-beam theory applies [10].

To solve these partial differential equations the Lagrangian coordinate formulation makes up the largest portion of the open literature. Initially these models are solved with a numerical semi-implicit finite differencing scheme [11]. The method is conditionally stable if the time step is carefully calculated based on the arc length and the tension. However, this method is not practical for industrial applications as the stability parameters vary dynamically. Therefore, unconditionally stable time integration methods are favoured. To improve the accuracy and stability of the solution Galerkin's finite element formulation and first-order Adams-Moulton time integration algorithm is implemented [12]. However, the multi-step integration implemented in Adams-Moulton method is computationally expensive. A variational finite element approach and the Newton-Raphson algorithm improved the computational feasibility of this method [13]. A static finite element method is proposed, to improve the deformation dependency of the boundary conditions and load points. This includes a predictor-corrector multi-step scheme formulation based on non-linear vectorial equilibrium formulation is proposed however, the method is computationally expensive and its stability is not discussed in the literature [14]. 
The increase in flexible riser installations required new formulations to overcome the simulation difficulties attributed to the large axial stiffnesses. Therefore, a 2D Cartesian in-extensible hybrid beam-column formulation is proposed for un-bonded flexible risers implementing a convected coordinate scheme; where the hybrid beam formulation is used to solve the flexible riser ill-conditioned system stiffness matrix problem. In addition, a local coordinate system is fixed to each element, to decouple the rigid body motion and strains. This is connected to the global Cartesian coordinate system via rotation angles allowing for large deflections [15]. The hybrid-beam element formulation is used in which the axial deformation and in-extensibility conditions are satisfied. The method uses non-linear programming techniques to apply the Lagrangian constraint and imposes the in-extensibility condition allowing for large deformations with convected axes. This method is extended to a 3D analysis [16] and implemented in a commercial riser three-dimensional non-linear finite element package Flexcom. The method uses Houbolt operator time integration which is shown to be suitable for the slow motion periods that flexible risers exhibit [9, 15]. Another time integration method is suggested, the Hilbert-Hughes-Taylor one step method which is unconditionally stable with linear systems and allows for variable time steps. The optimal time step is calculated automatically using the current step period parameter and after a few incremental steps the optimal value is chosen [17]. Recently a multi-scale element type for flexible risers is developed [18] which allows the stick/slip behaviour of flexible steel risers to be captured via a hybrid beam element user defined subroutine in Abaqus. However, this method mostly benefits un-bonded flexible riser analysis and doesn't provide additional information about the bonded cross-sections at the global riser scale since composite pipes are bonded and don't exhibit hysteresis like flexible risers.

The literature related to extensible formulations is sparse compared to the in-extensibility formulations. A nonlinear cable formulation is successfully solved with the finite element technique and New-mark's time integration method [19]. However, this method causes large errors in predicting the cable natural frequencies [20] as the lumped mass technique is implemented [21]. A full 3D extensible cable is derived in [22, 23], which is solved with an implicit second order box finite difference method [24] resulting in an unconditionally stable method that is discretized and centred in space and time and second order accurate. However, the method is developed for negligible flexural stiffness cables and requires the inverse of the system matrix, which causes a singularity in slack conditions. The method is expanded to include cables with considerable flexural stiffness and solved with Newton-Raphson method [25, 26] and later solved with relaxation method [27, 28].

Although the literature is rich with multiple extensible and in-extensible dynamic analysis methods, it lacks a comparison between the two methods especially to quantify the effect of composite riser cross-section on the calculated tensions and curvatures. 


\subsection{Mathematical formulation}

RiSim, a non-linear extensible, time-domain implicit Keller-box finite difference code is developed in FORTRAN based on the formulation derived in [24, 25]. The non-linear system of equations is solved with a modified Powell hybrid method. The method is suitable for calculating the local minima of complex functions without the need to calculate its derivatives [29]. RiSim is used to investigate the composite riser response and compared to a finite element in-extensible hybrid formulation implemented in the riser simulation package, Flexcom, which is based on the method developed in[17, 15, 16] to determine the accuracy of these models with the reduced axial stiffness exhibited by composite risers.

Riser design is performed in two stages for computational efficiency reasons. First the response of the entire riser is modelled under the influence of environmental and vessel loads. The effect of the maximum mean and low frequency vessel responses are modelled as a static step with an offset to the initial position of the vessel, while the high frequency dynamic excitations are captured within the vessel's RAOs. This dynamic time domain analysis is required to identify the critical riser responses and sections for ultimate and fatigue limits states. The second step is to build a detailed model of the critical cross-section. This analysis is performed to predict the stresses and strains in the laminate to optimise the layup. Outputs from the global analysis, the tensions and rotations at both ends of the critical sections are applied as boundary conditions to the cross-section model.

In this analysis the inline linear waves and sinusoidal hang-off excitation are applied to the riser system. The riser motion is assumed to occur in a 2D plan for simplicity. The governing dynamic equilibrium system is presented as a series of partial differential equations in 1 to 6 The derivation is based on the extensible non-linear Lagrangian partial differential equations representing the 2D riser boundary-value problem. Variables $u$ and $v$ are the velocities in the tangential and transversal directions $\hat{\mathbf{t}}$ and $\hat{\mathbf{n}}, \mathrm{T}$ is the tension, $S_{n}$ is the shear force in the transverse direction and $G_{b}$ is the curvature about the bi-normal direction $\hat{\mathbf{n}}$. $\Phi$ is the angle between the tangent and the horizontal. $m$ and $m a$ are the mass in air and the added mass per meter, $w_{0}$ is the apparent weight of the riser per meter. $d_{o}$ is the outer diameter of the riser. $E A$ and $E I$ are the axial and bending stiffnesses. $s$ is the Lagrangian, un-stretched coordinate, $v_{t r}$ and $v_{n r}$ are the tangential and transversal relative velocities, $C_{d t}$ and $C_{d n}$ are the tangential and transversal drag coefficients, $t$ is the time and $\rho$ is the sea water density, $v_{t c}, v_{n c}, v_{t w}$ and $v_{n w}$ are the current and wave tangential and transversal particle velocities.

The relationship between the rate of change of the velocities frame angle $\Phi$ with respect to time at each node as a function of tension variation and the total force balance along the riser in the tangential $\hat{\mathbf{t}}$ direction described in equation 1 . 


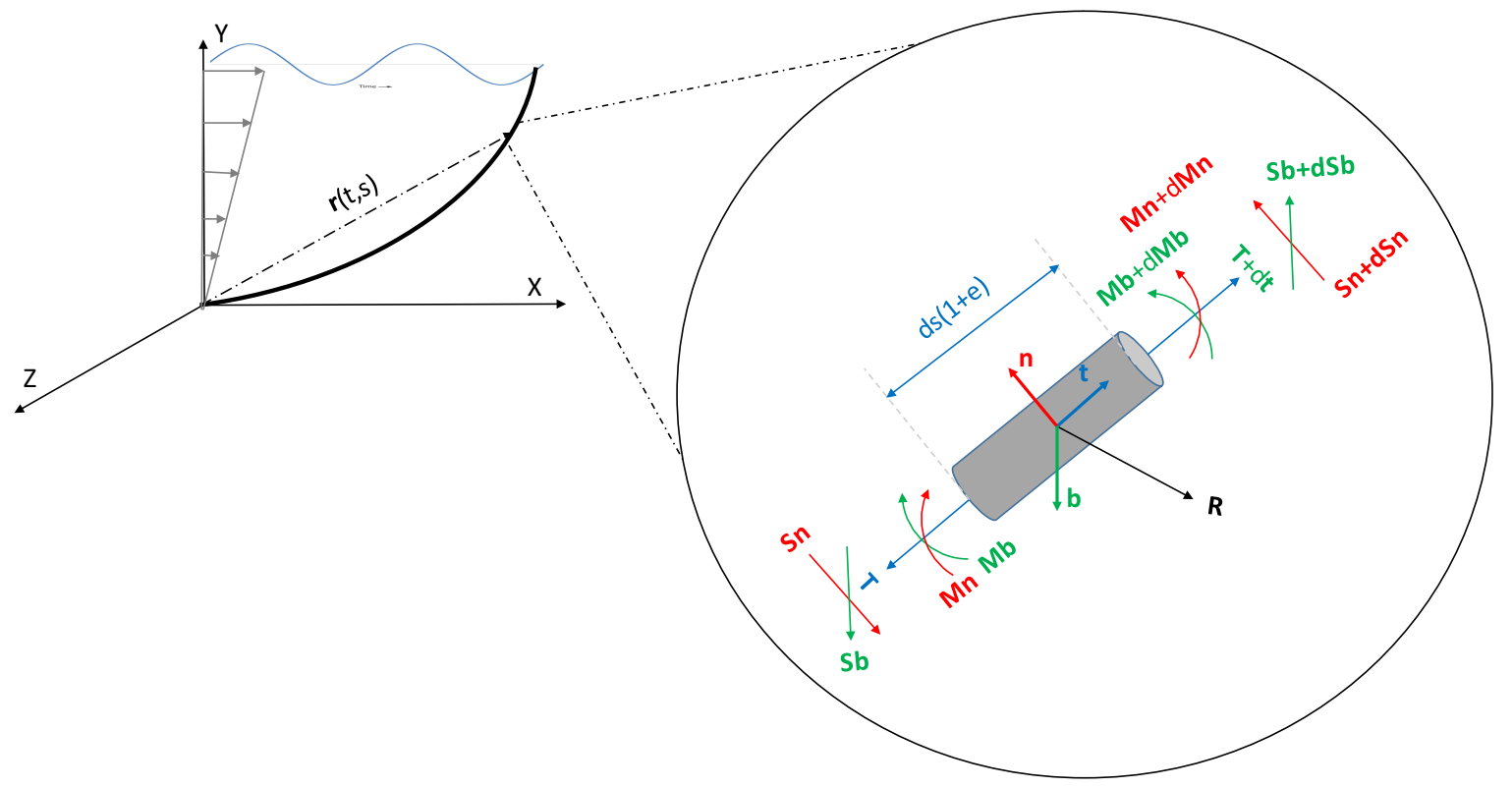

Figure 1: Riser element loads equilibrium diagram

$$
\begin{aligned}
& m\left(\frac{\partial u}{\partial t}-v \frac{\partial \Phi}{\partial t}\right)-\frac{\partial T}{\partial s}+S_{n} \Omega_{b}+w_{0} \sin \Phi \\
& +\frac{1}{2} \pi \rho d_{o} C_{d t} v_{t r}\left|v_{t r}\right| \sqrt{1+\left(\frac{T}{E A}\right)}=0 .
\end{aligned}
$$

and in transversal direction $\hat{\mathbf{n}}$ in 2 ,

$$
\begin{aligned}
& m\left(\frac{\partial v}{\partial t}+u \frac{\Phi}{\partial t}\right)+m_{a} \frac{\partial v_{n r}}{\partial t}-\frac{\partial S_{n}}{\partial s}-\Omega_{b} T+w_{0} \cos \Phi \\
& +\frac{1}{2} \rho d_{o} C_{d n} v_{n r}\left|v_{n r}\right| \sqrt{1+\left(\frac{T}{E A}\right)}=0 .
\end{aligned}
$$

The governing relationship between the temporal rate of change of the tension, as a function of axial stiffness, nodal velocities and curvatures, is described in equation 3 .

$$
\frac{\partial T}{\partial t}-E A\left(\frac{\partial u}{\partial s}-\Omega_{b} v\right)=0
$$


and the reference frame angle is shown in equation 4

$$
\left(1+\left(\frac{T}{E A}\right)\right) \frac{\partial \Phi}{\partial t}-\frac{\partial v}{\partial s}-u \Omega_{b}=0 .
$$

The coupled relationship between the axial and bending strains are provided in equation 5 .

$$
E I \frac{\partial \Omega_{b}}{\partial s}+S_{n}\left(1+\left(\frac{T}{E A}\right)\right)^{3}=0 .
$$

While the definition for the curvature as a function of the reference frame angle along the riser is provided in equation 6

$$
\frac{\partial \Phi}{\partial s}-\Omega_{b}=0 .
$$

The tangential and transverse relative velocities, when the wave and current are applied, are given by equations 7 and 8 ,

$$
\begin{aligned}
& v_{t r}=u-v_{t c}-v_{t w}, \\
& v_{n r}=v-v_{n c}-v_{n w} .
\end{aligned}
$$

\subsection{Boundary conditions}

The 2D riser behaviour is governed by six, first order partial differential equations. The finite difference discretisation leads to $n-1$ equations and the missing 6 equations to solve the system are given by the equal number of boundary conditions given by equations 9

At the touch-down point the boundary conditions are given by equation 9

$$
G_{b}^{0}=0, u^{0}=0, v^{0}=0,
$$

and the boundary conditions in the case of wave loading and pinned hang-off node are defined in equation 10 .

$$
G_{b}{ }^{N-1}=0, u^{N-1}=0, v^{N-1}=0 .
$$


Assuming a sinusoidal excitation at the hang-off node, the boundary conditions are given by equation 11 .

$$
G_{b}{ }^{N-1}=0, u^{N-1}=0, v^{N-1}=5.0 \sin (\omega t) .
$$

\subsection{The Keller-box method}

The Keller-box numerical scheme is an implicit method, designed to solve first order parabolic partial differential equations in time and space [30]. The solution is second order accurate and simple to implement for large numbers of coupled PDEs. The method requires all the PDEs to be reformulated in first order form before discretisation.

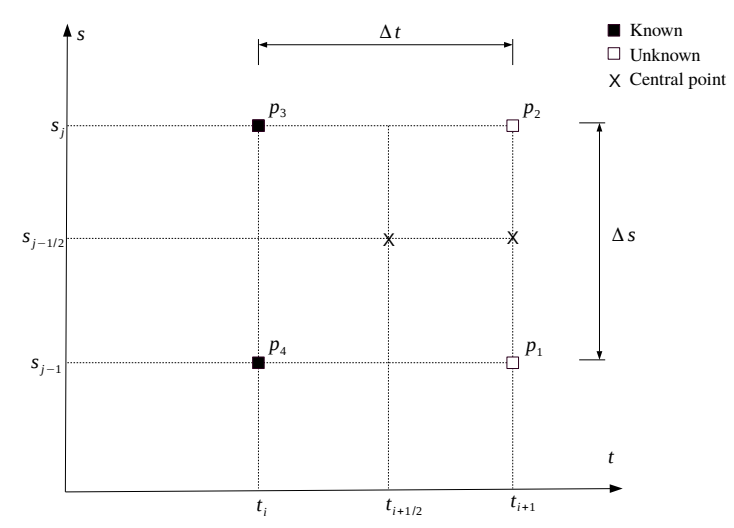

Figure 2: Keller-box method finite difference grid

The algorithm requires discretisation in space and time that is chosen at the central point $\left(t_{i+1 / 2}, s_{j-1 / 2}\right)$, where $i$ and $j$ represent the current time and spatial node. The discretization is performed between the points $P_{1}, P_{2}, P_{3}$ and $P_{4}$ as shown in figure 2. These points represents the function evaluation at either the previous time, the next time or spatial step. Let $Y$ be the unknown variable and then the central differencing of the temporal derivative at the centre point is determined by, fixing the spatial coordinate while the temporal derivative is replaced by equation 12 .

$$
\begin{aligned}
\frac{\partial Y}{\partial t} & =\frac{Y_{j-1 / 2}^{i+1}-Y_{j-1 / 2}^{i}}{\Delta t_{i}}+O\left(h^{2}\right) \\
& =\frac{Y_{j}^{i+1}+Y_{j-1}^{i+1}-Y_{j}^{i}-Y_{j-1}^{i}}{\Delta t_{i}}+O\left(h^{2}\right) .
\end{aligned}
$$


Similarly, the spatial derivative is replaced by equation 13 .

$$
\begin{aligned}
\frac{\partial Y}{\partial s} & =\frac{Y_{j}^{i+1 / 2}-Y_{j-1}^{i+1 / 2}}{\Delta s_{i}}+O\left(h^{2}\right) \\
& =\frac{Y_{j}^{i+1}+Y_{j}^{i}-Y_{j-1}^{i+1}-Y_{j-1}^{i}}{\Delta s_{i}}+O\left(h^{2}\right) .
\end{aligned}
$$

By neglecting the higher order terms and expanding equations 12,13 the central point is given by the expressions in equation 14

$$
t_{i+1 / 2}=\frac{1}{2}\left(t_{i+1}+t_{i}\right), s_{j-1 / 2}=\frac{1}{2}\left(s_{j}+s_{j-1}\right) .
$$

\section{Riser model benchmarking}

The static and dynamic responses of the riser are benchmarked using the in-house developed algorithm RiSim and FE commercial package Flexcom. An 11 inch composite catenary riser is investigated with a comparison of when it is unpressurised and with a 200 bar internal pressure. The riser properties and environmental data are listed in table

1. including the maximum wave height $H_{\max }$ and corresponding peak wave period $T_{p}$. A wave train that originates at a location above the global axes origin, on the seabed, that is located at the mid-water line propagates in the positive horizontal axes direction. The seabed is intentionally not modelled in this study to isolate the effects of extensibility on the riser response. The structural damping depends on the type of structure and the material; this is made more complex when considering composite materials as they exhibit variable damping properties which are dependent on the natural frequency of each vibration mode. Damping tests are required to quantify these variable damping values, as assuming variable damping can be misleading. Therefore, riser analysis is performed without structural damping as a base case [31, 32]. 


\begin{tabular}{lr}
\hline Parameter & Value \\
\hline Water depth $(d)[m]$ & 400.00 \\
Total riser length $(S)[m]$ & 487.43 \\
Bending stiffness $(E I)\left[\mathrm{Nm}^{2}\right]$ & $49.52 \mathrm{E}+06$ \\
Axial stiffness $(E A)[N]$ & $37.23 \mathrm{E}+09$ \\
Mass in air empty $(m)[\mathrm{kg} / \mathrm{m}]$ & 163.85 \\
Internal fluid density $\left(\rho_{f}\right)\left[\mathrm{kg} / \mathrm{m}^{3}\right]$ & 1025.0 \\
Tangential drag coefficient $\left(C_{D t}\right)$ & 0.05 \\
Normal drag coefficient $\left(C_{D n}\right)$ & 1.2 \\
Outer diameter $\left(d_{o}\right)[m]$ & 0.324 \\
Internal diameter $\left(d_{i}\right)[m]$ & 0.28 \\
Maximum wave height $\left(H_{m a x}\right)[m]$ & 18.6 \\
Wave period $\left(T_{p}\right)[s]$ & 14.9 \\
Hang-off horizontal coordinate $\left(X_{H O}\right)[m]$ & 224.7 \\
Hang-off vertical coordinate $\left(Y_{H O}\right)[m]$ & 400.00 \\
Internal Pressure $($ bar $)$ & $0 / 200$ \\
\hline
\end{tabular}

\subsection{Static comparison of extensible and in-extensible methods}

A comparison between the tensions and bending moments predicted by the extensible and in-extensible formulations are shown in figure 3 for the tension and figure 4 for the resultant bending moment. A visual check of the figures 3 -4 infers that both methods predict tension and bending moments that are indistinguishable from each other. That is because the static tension and bending moments demonstrate a negligible percentage differences of less than $0.02 \%$ for the maximum static tension, while the maximum bending moment demonstrates a $0.09 \%$ difference which shows that both methods are predicting similar static solution. 


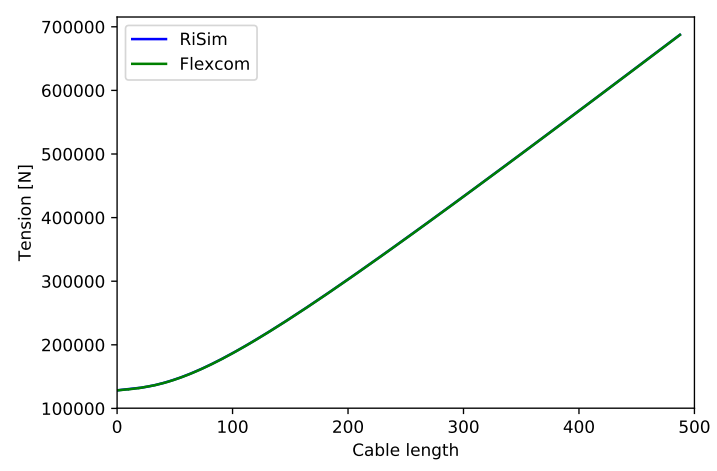

Figure 3: Effective tension along riser length

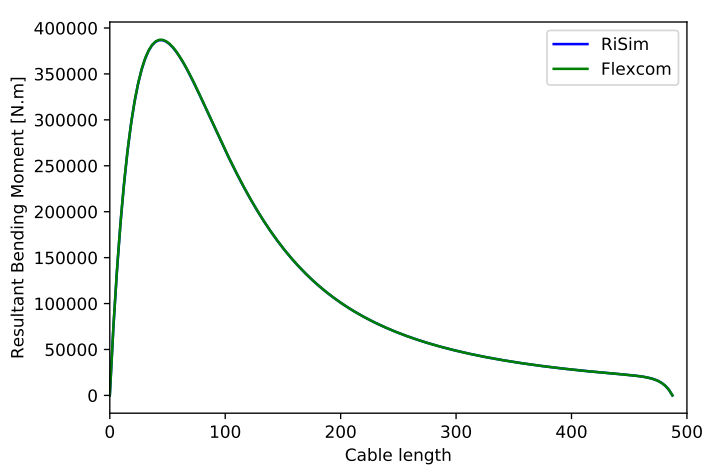

Figure 4: Resultant bending moment along riser length

\subsection{Dynamic comparison of extensible and in-extensible methods}

\subsubsection{Comparison in Regular waves}

Dynamic riser analysis is performed by applying regular waves to the riser system. This test is used to verify the regular wave algorithm used in RiSim before applying the top-side excitation. The waves data are chosen based on the extreme conditions from a 100 year return period extreme conditions that typically occurs at west of the Shetlands. A good match is reported between the two methods as documented in table 2 for an extreme wave height and table 3 for a moderate wave height. When applying an extreme wave that has 34.5 meters wave height and a 17.5 seconds wave period, the maximum percentage difference between the two methods along the length of the risers is at the minimum tension with a value of $0.65 \%$, as shown in table 2 However, the maximum percentage difference reduces to $0.06 \%$ when a moderate wave is applied with 18.6 meters wave height and 14.9 seconds wave period, table 3 Showing that when an extreme wave condition with a 100-year return period is applied, the differences between the two models are negligible with less than $1 \%$ maximum error. This gives confidence in the regular wave algorithm implemented in the RiSim code.

Table 2: 100 year extreme wave conditions-pinned model

\begin{tabular}{lrrr}
\hline & \multicolumn{3}{c}{ Hmax $=34.5 \mathbf{m}$} \\
Tp=17.5s & \\
\cline { 2 - 3 } Parameter & Flexcom & FD & D\% \\
\hline Max Tension $[k N]$ & 709.25 & 709.83 & $0.08 \%$ \\
Min Tension $[k N]$ & 671.92 & 667.52 & $-0.65 \%$ \\
Max Curvature $\left[m^{-1}\right]$ & 0.0086 & 0.0086 & $0.0 \%$ \\
\hline
\end{tabular}

Despite the similarity in results, the FE in-extensible solution experiences some numerical instabilities during the first wave period and a number of smaller numerical instabilities are exhibited. However, while the extensible finite difference solution converges immediately to the final response and doesn't experience high frequency spurious numerical oscillations. Looking beyond the first wave period, the comparison between the two methods is shown in figure 5 which shows the time-trace of the 


\begin{tabular}{lrrr}
\hline & \multicolumn{4}{c}{ Hmax=18.6 } \\
\cline { 2 - 4 } Tp=14.9 & \\
\cline { 2 - 4 } Parameter & Flexcom & FD & D\% \\
\hline Max Tension $[k N]$ & 694.37 & 694.84 & $0.07 \%$ \\
Min Tension $[k N]$ & 680.4 & 679.99 & $-0.06 \%$ \\
Max Curvature $\left[m^{-1}\right]$ & 0.0084 & 0.0084 & $0.0 \%$ \\
\hline
\end{tabular}

hang-off node tension performed in both Flexcom and RiSim with 3 meter element lengths. Figure 5 shows that elastic waves are excited in addition to the transverse waves, and these are only captured by the extensible riser formulation. For typical composite risers, which are likely to have axial and flexural stiffnesses separated by only three orders of magnitude, elastic waves can be excited and the behaviour can be captured using the extensible formulation implemented in RiSim.

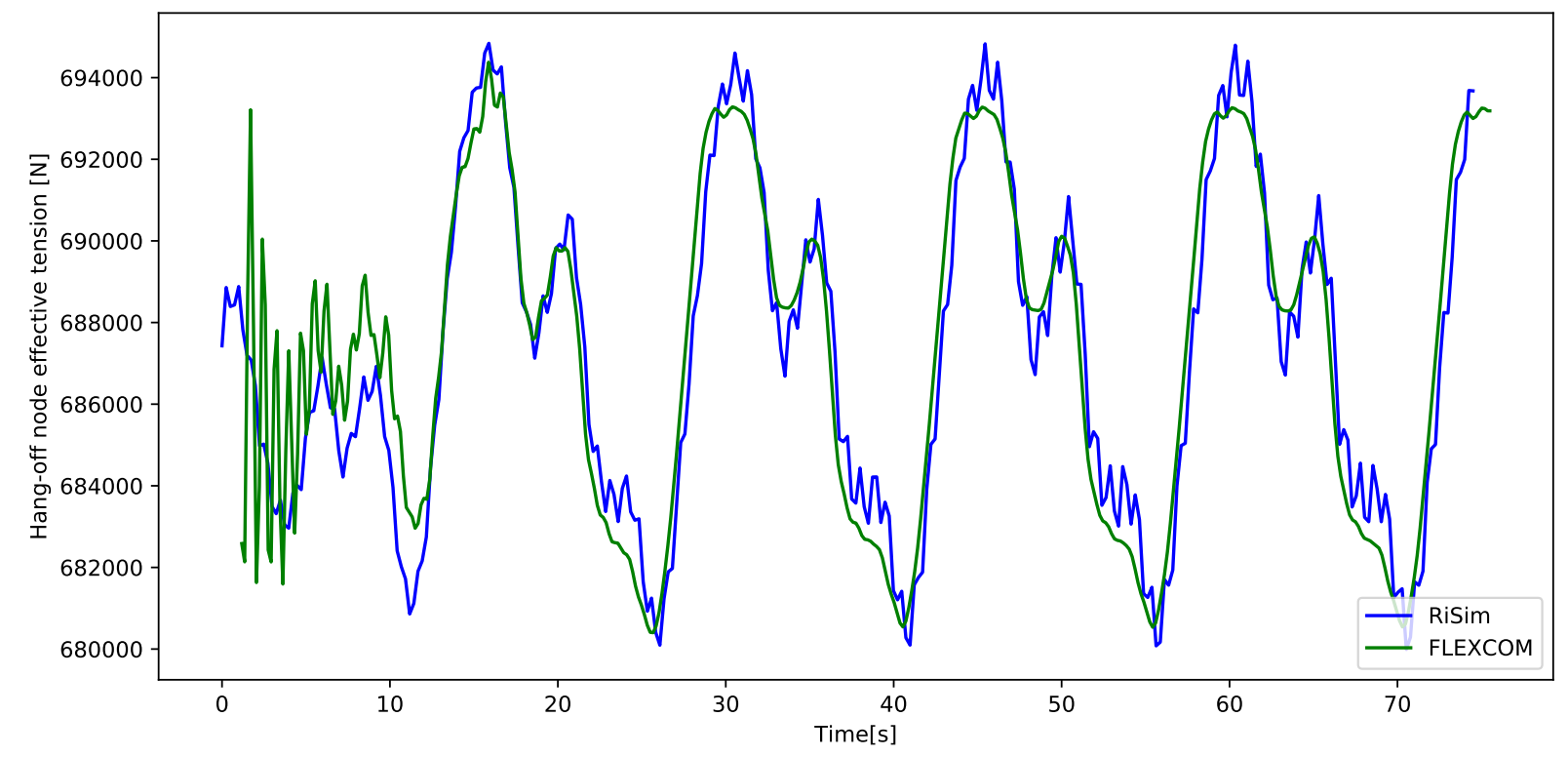

Figure 5: Hang-off effective tension

\subsubsection{Regular waves with top-side excitation}

Two types of sinusoidal excitations are applied to replicate a general range of top-side excitations in the surge direction: an excitation with angular frequency that is in phase with the wave train and another 180 degrees out of phase. The excitation is applied to the hang-off node of a 200 bar pressurised and unpressurised riser in the transverse direction in addition to the regular waves. Using equation 11 a sinusoidal velocity is applied in the normal direction $\vec{n}$ to the hang-off node. The corresponding Cartesian time traces of the horizontal and vertical coordinates are extracted and applied to the FEA in-extensible model as a displacement boundary condition applied to the hang-off node. 
Both methodologies match well for the in-phase wave with the top-side excitations using the hang-off tension time-trace. A maximum difference of less than $0.5 \%$ is observed after the inextensible method has stabilised beyond the first wave period. The results from the 180 degree phase shift also exhibits a close behaviour with a maximum $1 \%$ percentage difference after the $\mathrm{FE}$ solution converges to the steady state solution after three wave periods, this is shown in figure 6 Although the variation between both methods is within the maximum documented percentage difference between dynamic analysis methods in the literature [33], figure 6 illustrates this difference for the pressurised riser. It is observed, for both the pressurised and unpressurised cases, that the RiSim Finite Differencing algorithm converges immediately to the final steady state system response while the finite element solution experiences higher order oscillations that are sensitive to the solutions convergence tolerance.

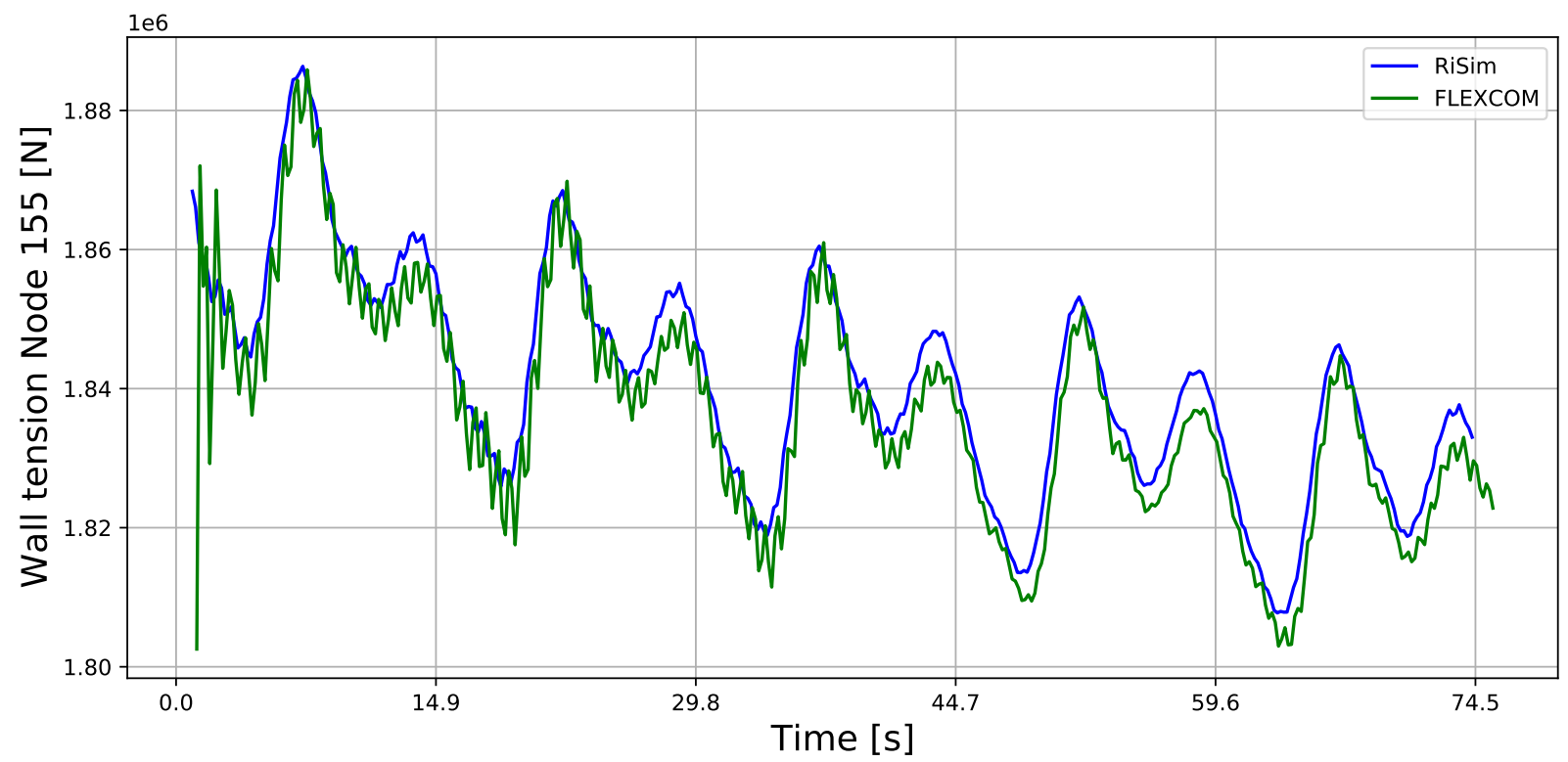

Figure 6: Hotspot wall tension time-trace

The in-extensible method experiences 3 times the number of cycles for the tension ranges up to $20 \mathrm{kN}$ compared to the extensible formulation, figure 7 The number of cycles reduces to 2 times larger if the first 3 wave periods are ignored from the in-extensible analysis. This implies that often the first 3 wave periods should be avoided when using in-extensible methods and a longer simulation period is required for reliable results. It is not likely that the high order oscillations have a large effect on the total fatigue life of the riser, however either human or automated filtering process is required to avoid these numerical instabilities. This problem is not experienced with the extensible formulation.

The hybrid element formulation is a probable cause for such instabilities. It is implemented in the in-extensible software where the in-extensibility condition is enforced artificially, in which the axial strain is decoupled and interpolated separately. This type of mixed field variable formulation is reported to cause spurious numerical errors if the interpolation function used with the axial force is not the same order as the strain $\epsilon$. This can cause a second kind errors in the solution even for arbitrarily small meshes 
[15]. The extensible method solved with Keller-box scheme finite difference is demonstrated to be stable and converges in fewer time steps to the final system steady state response while remaining consistent to the results from the in-extensible model.

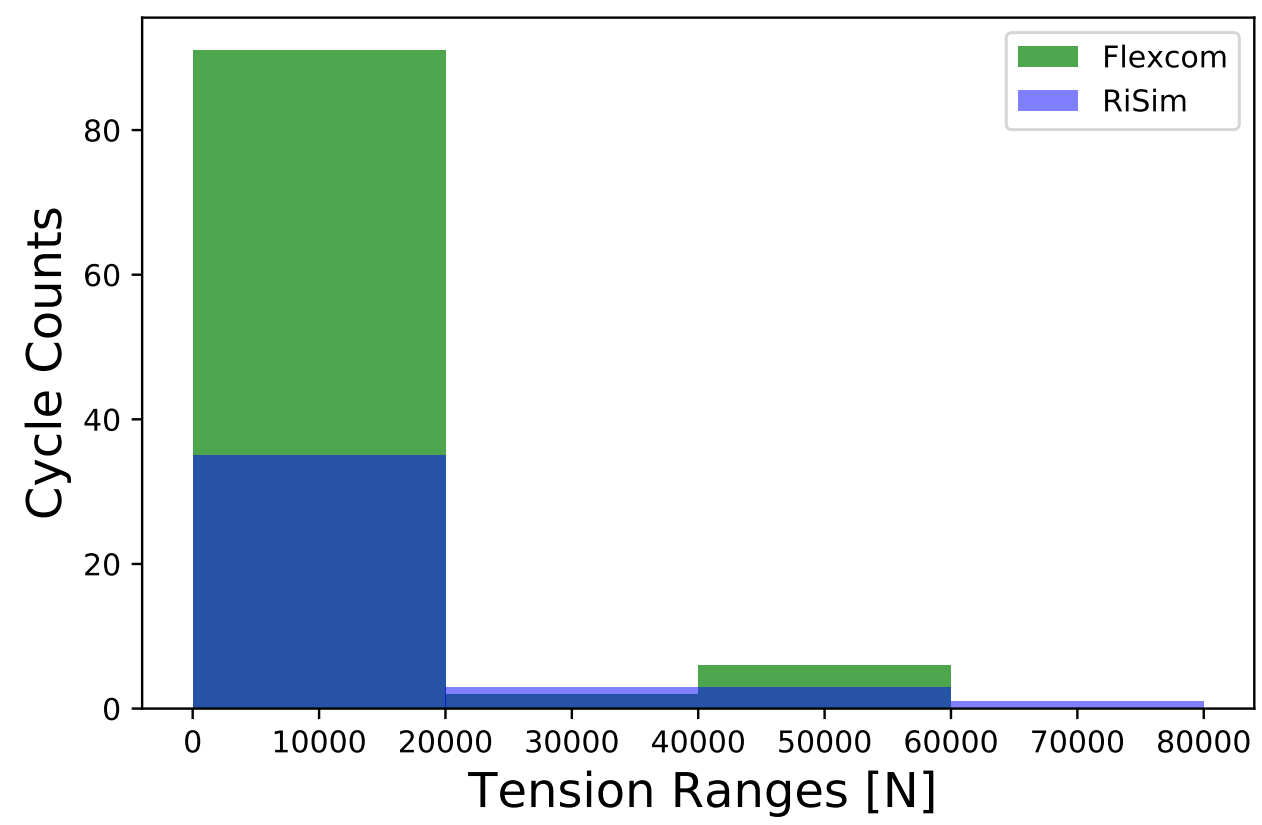

Figure 7: Tension range Vs Cycle count

To investigate the phenomena further, the tension time-traces high frequency cycles are counted using the rain-flow method applied to the extensible and in-extensible results. It is found that for the riser system analysed the ratio of the observed high frequency oscillations average amplitude to the top tension high frequency cycle amplitude is close to $1.5 \%$, therefore cycle counting is performed at $1.5 \%$ of the top tension range. The oscillations are calculated using rain-flow counting algorithm to investigate this phenomena.

To assess the effect of riser cross-section design on the top tension high frequency cycles. A non-dimensional parameter $\lambda$ is introduced, defined in equation 15 The parameter is suitable for investigating the relationship between the in-extensible formulation and the high frequency oscillations that are observed, since it is used to investigate the cross-over phenomena of the cable elastic and transverse natural frequencies in [34]. Four riser cross-sections are assessed, where the internal diameter of the riser is kept constant while increasing the outer diameter. The corresponding cross-sectional properties are estimated as shown in table 4 and used in the analysis. The analysis performed in section 3.2 .2 is repeated with the cross-sections and the results are is shown in figure 8

Figure 8 demonstrates the relationship between the parameter $\lambda$, varied as multiples of $\pi$, in comparison to the number of cycles from the rain-flow counting of the high frequency tension cycles with amplitude $1.5 \%$ of the average tension. A curve is fitted to the data points to demonstrate the trend. It is found that both extensible and in-extensible formulations follow a closely related trend with respect to $\lambda$, however the effect of the high order oscillations observed in the in-extensible commercial code is 
Table 4: Riser Load Cases

\begin{tabular}{lrrrr}
\hline Parameters & LC1 & LC2 & LC3 & LC4 \\
\hline Internal diameter $[\mathrm{m}]$ & 0.28 & 0.28 & 0.28 & 0.28 \\
Outer diameter $[\mathrm{m}]$ & 0.35 & 0.42 & 0.49 & 0.55 \\
Thickness $[\mathrm{m}]$ & 0.03 & 0.07 & 0.10 & 0.14 \\
Bending Stiffness (EI) $\left[\mathrm{Nm}^{2}\right]$ & $3.87 \mathrm{e}+07$ & $9.27 \mathrm{e}+07$ & $1.78 \mathrm{e}+08$ & $3.06 \mathrm{e}+08$ \\
Axial Stiffness $(\mathrm{EA})[\mathrm{N}]$ & $3.12 \mathrm{e}+09$ & $5.90 \mathrm{e}+09$ & $9.07 \mathrm{e}+09$ & $1.27 \mathrm{e}+10$ \\
Mass in air $[\mathrm{kg} / \mathrm{m}]$ & 116.70 & 216.22 & 329.79 & 460.02 \\
$\lambda[-]$ & $16.33 \pi$ & $18.46 \pi$ & $20.19 \pi$ & $17.19 \pi$ \\
\hline
\end{tabular}

demonstrated in figure 8 where the average number of cycles is between 2 to 8 times higher than predicted by the extensible code.

The low amplitude, high frequency, spurious oscillations are only observed in the in-extensible tension time-traces and don't appear in the curvature time-trace. This suggests that the behaviour is related to the interpolation function implemented in the hybrid element formulation and so the extensible formulation is favoured for composite cross-sections.

$$
\lambda=\sqrt{\frac{\left(w_{0} S\right)^{2} E A_{0}}{T_{\text {horz }}^{3}} \cos \Phi_{a}}
$$

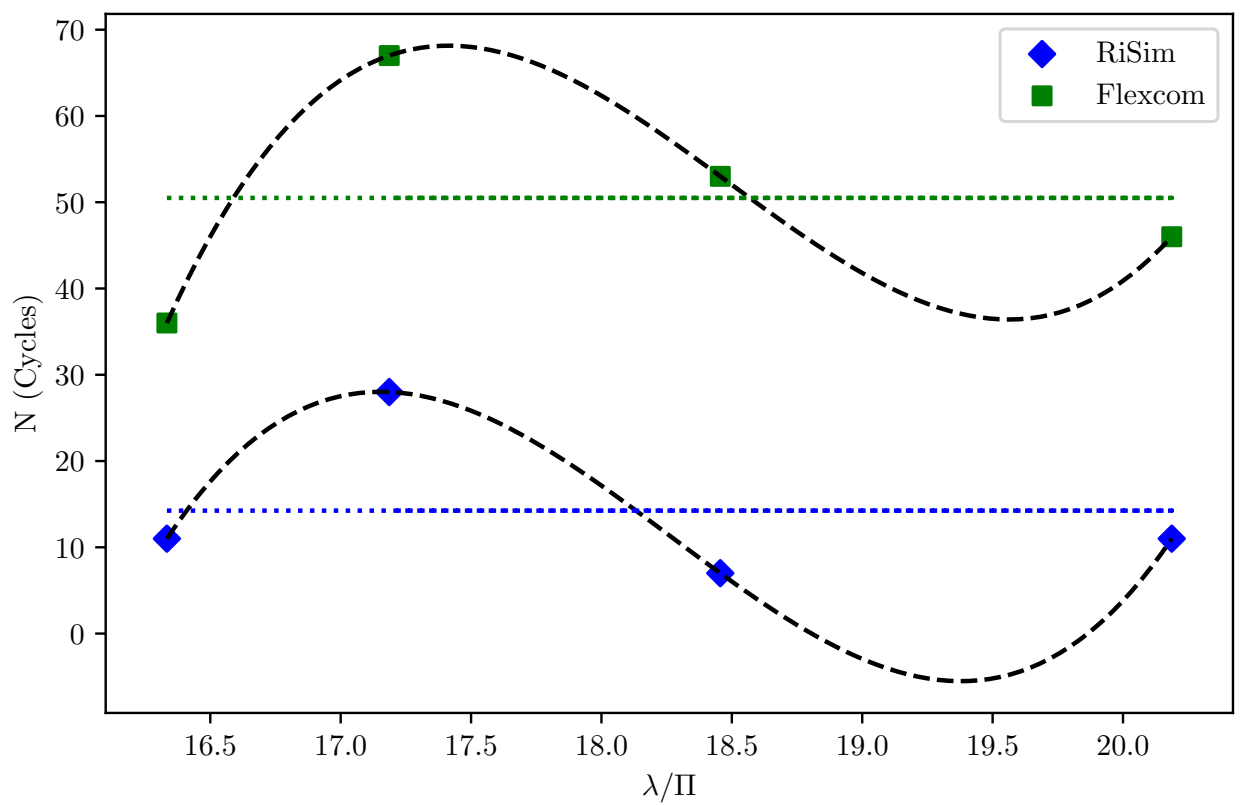

Figure 8: Number of Cycles $\mathrm{N}$ at $\Delta \mathrm{T}=1.5 \%$ static tension at hot-spot 


\section{Composite pipe numerical model}

Global riser analysis is required to estimate the critical section of the riser, the 'hot-spot'. The tension and curvatures timetraces are then imposed as boundary conditions to the composite pipe model. However, there is a gap in the composite pipe modelling literature for offshore applications where it is not clear what elements can be used to accurately model the laminate stresses. Therefore further studies are required to provide higher confidence level in the modelling methods.

Various element types are developed to model composites: equivalent single layer shells, layer-wise continuum shell and 3D continuum solid elements [35. The suitability of these element depends on the pipe thickness to bending radius ratio. A pipe tends to behave like a thick shell if the ratio between its bending radius and thickness is lower than a specific threshold. However, there is no clear separation in the literature between thin and thick shells for cylindrical pipe applications. Metallic pipes under uniform bending are considered thick and modelled with brick elements as long as the bending radius to thickness ratio is below a factor of 10, while solid continuum elements become inaccurate with larger ratios i.e ratios beyond 25 [36]. 3D solid elements are used for the laminate layup and thickness optimisation by constraining one displacement and applying pure tension on the other end, however the study is limited to laminates under tension load[37]. Homogenisation is used to reduce the computational burden, especially for thick composites, where homogenised elastic constants are derived based on an asymptomatic expansion. The homogenised elastic constants is beneficial when it depends on the stacking sequence, that is often the case with larger thicknesses and small radii. The results obtained from homogenisation methods of thin composites are often identical to single layer theories [38].

To assess the suitability and computational efficiency of shell, continuum shell and solid elements for composite riser modelling, the loads that are predicted by the global model are imposed as boundary conditions to a composite pipe model which is built in ABAQUS finite element packages. A comparison is made between the following elements: quadratic composite shell $S 8 R$, continuum composite shell $S C 8 R$, linear solid composite $C 3 D 8 R$ and quadratic solid composite $C 3 D 20 R$. The results are compared to the homogenization and FE methods that are reported in [38].

\subsection{FEA model definition}

A 22-layer carbon/epoxy composite riser is chosen based on the geometry tabulated in [38]. The composite riser consists of (from inner to outer layers), a titanium internal liner, composite layers with a layup $[0 / 90 / 45 /-45]_{5}$ and a polymer outer sheath. The cross-section design and stacking sequence are illustrated in figure 9 The pipe bonded cross-section dimensions and lay-up are listed in table 5, where contact is not explicitly considered. The titanium liner, composite plies and outer sheath material properties used in the study are in table 6

Where $E_{1}$ and $E_{2}$ are the Young's moduli along the fibre and in the transverse direction respectively; $v_{12}, v_{23}, v_{13}$ are the Poisson's ratios; $G_{12}$ and $G_{13}$ are the in-plane shear moduli and $G_{23}$ is the transverse shear modulus. For failure prediction: $X_{T}$ and $X_{C}$ are the longitudinal tensile and compressive strengths; $Y_{T}$ and $Y_{C}$ are the transverse tensile and compressive strengths and $S$ is the in-plane shear strength. 
Table 5: Composite riser cross-sectional data

\begin{tabular}{lr}
\hline Parameters & Value \\
\hline Internal diameter $[\mathrm{m}]$ & 0.28 \\
Outer diameter $[\mathrm{m}]$ & 0.31432 \\
Pipe length $[\mathrm{m}]$ & 10.0 \\
Liner thickness $[\mathrm{m}]$ & 0.005 \\
Outer sheath thickness $[\mathrm{m}]$ & 0.002 \\
Composite layers thickness $[\mathrm{m}]$ & 0.01016 \\
\hline
\end{tabular}

Table 6: Composite riser material properties

\begin{tabular}{lll}
\hline Liner & Composite & Outer sheath \\
(Titanium) & ply & (Polymer) \\
\hline$E=120.0$ & $E_{1}=135.0$ & $\mathrm{E}=3.0$ \\
$v=0.33$ & $E_{2}=8.0$ & $v=0.40$ \\
$E_{3}=8.0$ & \\
$G_{23}$ & $=2.7$ & \\
$G_{13}$ & $=3.8$ & \\
$G_{12}$ & $=3.8$ \\
$v_{23}$ & $=0.49$ \\
$v_{13}$ & $=0.27$ \\
$v_{12}$ & $=0.27$ \\
$X_{T}$ & $=2.45$ \\
$X_{C}$ & $=1.57$ \\
$Y_{T}$ & $=0.07$ \\
$Y_{C}$ & $=0.133$ \\
$S$ & $=0.098$ \\
\hline
\end{tabular}

[1] All moduli are in GPa

The boundary conditions and reference points are illustrated in figure 10 Both ends of the pipe are constrained using multipoint constraint to reference points that lie on the pipe neutral axis. The reference points are designated RP1 and RP2 and are located in the plane perpendicular to the pipe ends. The boundary conditions and reference points are illustrated in figure 10 Reference points RP1 and RP2 are allowed to rotate freely but RP1 is constrained in the translational X, Y and Z directions. RP2 is free to move in the longitudinal $\mathrm{Z}$ direction. Boundary conditions and loads are applied to the reference points and transferred via 


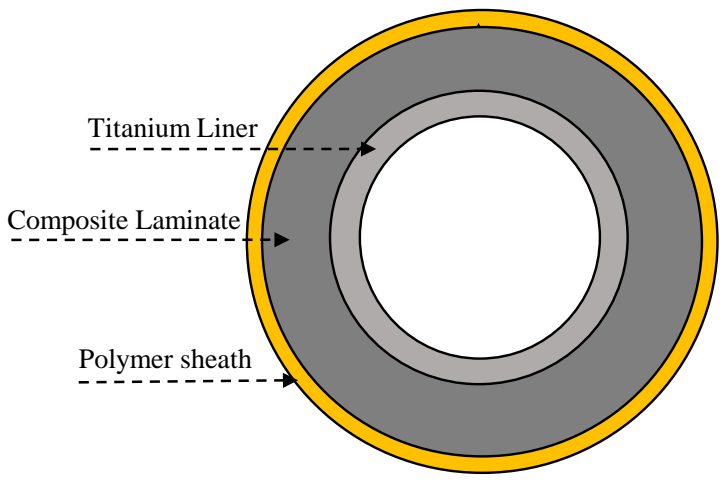

Composite riser cross-section

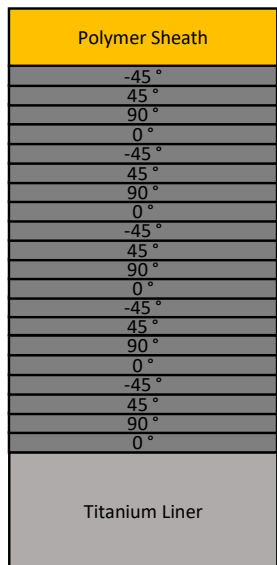

Stacking Sequence

Figure 9: Composite riser cross-section design and stacking sequence

the multi-point constraint to the circumference, to assure even distribution of loads. The boundary conditions and reference points are illustrated in figure 10

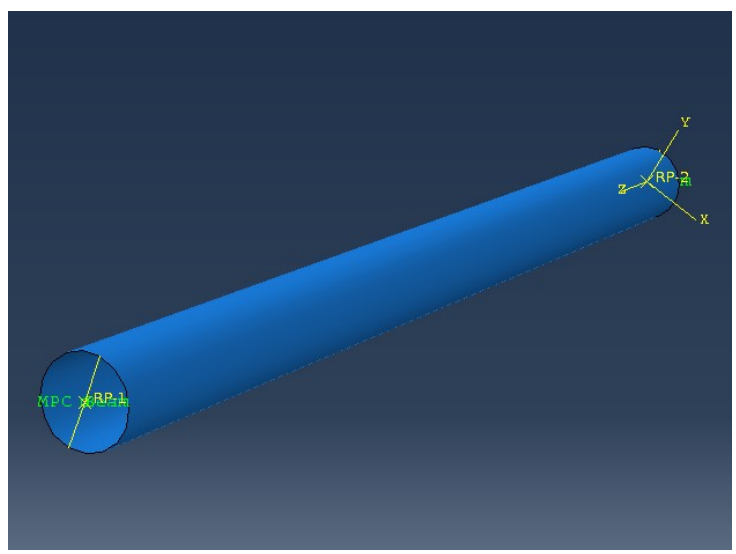

Figure 10: Pipe model MPC constrain 
Table 7: FEA element type comparison

\begin{tabular}{lrrrr}
\hline Element type & nodes & elements & D.O.F & CPU time[s] \\
\hline C3D20R & 168240 & 24000 & 506172 & 705.27 \\
C3D8R & 48098 & 24000 & 144876 & 260.37 \\
SC8R & 48098 & 24000 & 144876 & 43.95 \\
S8R & 66090 & 22000 & 396540 & 83.85 \\
S4R & 22046 & 22000 & 132276 & 27.51 \\
\hline
\end{tabular}

\section{Composite pipe benchmarking}

The benchmarking cases are chosen to assess the suitability and computational efficiency of shell, continuum shell and solid elements for composite riser modelling. Stress distributions across the laminate thickness are investigated, under axial and bending moment loading conditions. Four models are developed and the results are compared to the homogenization and FE methods that are reported in [38]. Figures 11 , 12 shows the longitudinal and hoop stresses $\sigma_{z}$ resulting from axial force of $1 \mathrm{kN}$ and bending moment load of $1 \mathrm{kN} . \mathrm{m}$. The model results obtained from the static FE analysis are found to match the stresses obtained by the homogenization method in [38]. All the elements are reported to predict the stress distribution for the tension and bending cases within a $5 \%$ difference. It's found that layers with fibre angles of $90^{\circ}$ and $\pm 45^{\circ}$ experience higher hoop stresses, compared to the Titanium liner, outer polymer sheath and $0^{\circ}$ fibre plies. Maximum axial stresses appear in the Titanium liner and $0^{\circ}$ composite plies as shown in figure 11 and 12

A 10 meter pipe with the properties shown in tables 5 and 6 is loaded with a combined $1 \mathrm{kN}$ axial force and a $1 \mathrm{kN}$.m moment at both reference points RP1 and RP2. The solid quadratic brick element $C 3 D 20 R$ is taken as a reference in this study to quantify the relative percentage difference of each element type. Table 7 provides the total number of nodes, elements, degrees of freedom and CPU time required for this analysis.

Figures 11,12 show the maximum percentage differences in stress of $4 \%$, which are exhibited by the linear and quadratic shell $S 4 R$ and $S 8 R$ elements. Lower percentage differences are observed for the $S C 8 R$ continuum shells and the linear $C 3 D 8 R$ brick element which shows the lowest difference. However, the $S C 8 R$ predicts hoop stresses closer to the quadratic solid $C 3 D 20 R$ than the $C 3 D 8 R$ brick element. In general the $S C 8 R$ elements provide an acceptable prediction accuracy compared to it's computational cost which is demonstrated in table 7 Therefore, the continuum shell element $S C 8 R$ is recommended to model composite risers as it provides the optimum computational cost to accuracy followed by $S 8 R$ element. 


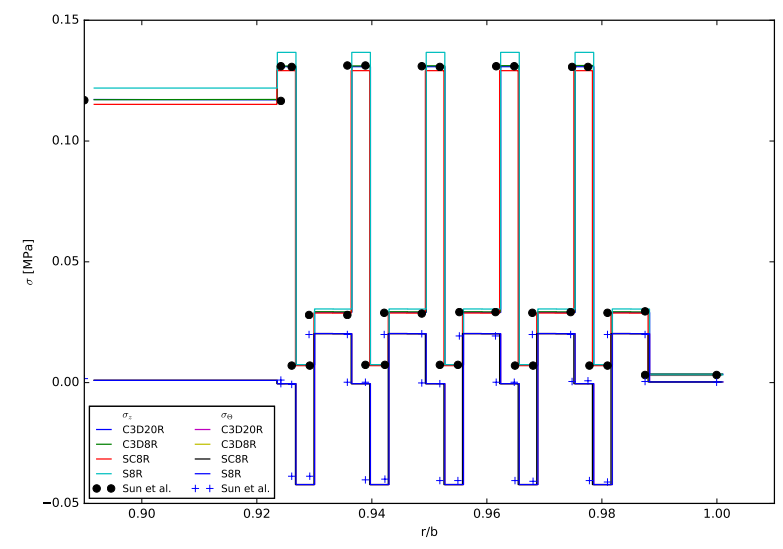

Figure 11: Stress distributions under axial force

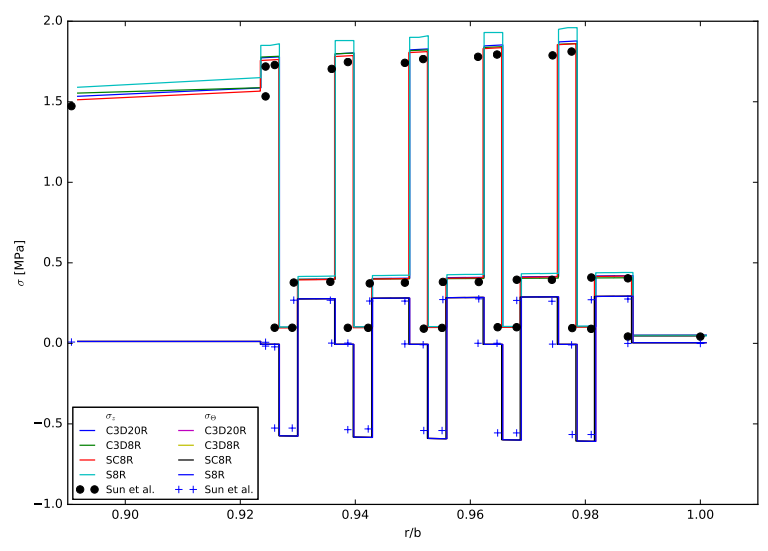

Figure 12: Stress distributions under bending moment

\section{Riser-pipe coupled dynamic analysis}

The coupled responses between the global and local responses are compared when using the extensible and in-extensible formulations. First the dynamic global analysis results that are described in section 3.2 .2 are used to identify the hot-spot. Then, a finite element model of the pipe is built for this critical section to investigate the effect of the global loading time-traces predicted by the global models on the composite laminate stresses. The extensible and in-extensible models are used to predict the global riser behaviour, where a top-side sinusoidal motion is applied in the surge direction and the environmental loads are modelled using linear waves according to the methodology described in section 2 in addition to the parameters in table 1 The rotations and tension time-traces are extracted from the global models and used as boundary conditions for the finite element continuum shell, composite pipe model. The finite element implicit time domain analysis is performed using the continuum shell elements, $S C 8 R$. The simulation is five wave periods long with a total time for the simulation of 74.5 seconds. The hot-spot is identified based on the highest tension and curvature standard deviations. Tensions and curvatures time-traces are the output of the riser analysis, which are applied to the composite pipe model.

A failure criteria is required, in order to quantify the effect of the global methods on the failure of the composite riser at the pipe level. The Tsai-Wu failure criterion is selected, which is a commonly used criterion to predict the first-ply failure and it is recommended as one of the more robust failure theories by the World Wide Failure Exercise. The first-ply is predicted to fail if the left-hand side of equation 16 is equal to, or higher than, unity. Where the terms $\sigma_{1}, \sigma_{2}$ and $\tau_{12}$ are the fibre longitudinal stress, the stress in the normal to fibre direction and shear stress in the ply; $X_{t}$ and $Y_{t}$ are the longitudinal and transverse tensile strengths; while $X_{c}$ and $Y_{c}$ are the compressive strengths and; $S_{12}$ is the in-plane shear strength. $F_{1}$ is a failure coefficient that is given by equation 17 Composite risers are designed with large safety factors and therefore failure is not expected with the riser properties 
used in this study. However, the criteria is used here to quantify the effect of both the extensible and in-extensible global models on the Tsai-Wu failure index at the hot-spot location.

$$
\begin{gathered}
\left(\frac{\sigma_{1}}{X_{t} X_{C}}\right)^{2}+\left(\frac{\sigma_{2}}{Y_{t} Y_{C}}\right)^{2}+\left(\frac{1}{X_{T}}-\frac{1}{X_{C}}\right) \sigma_{1}+\left(\frac{1}{Y_{T}}-\frac{1}{Y_{C}}\right) \sigma_{2}+\left(\frac{2 F_{1} 2 \sigma_{1} \sigma_{2}}{\sqrt{X_{T} X_{C} Y_{T} Y_{C}}}\right)+\left(\frac{\tau_{12}}{S_{12}}\right)^{2}=1 \\
F 1=\frac{1}{X_{t}}-\frac{1}{X_{c}}
\end{gathered}
$$

The boundary conditions are described in section 2.3 The tension time-traces are extracted from the riser beam model simulation and applied to node RP2 as a point force via the amplitude module in ABAQUS while the rotation angle time-traces are applied to both RP1 and RP2 reference points as boundary conditions.

\subsection{Dynamic results}

The results shows that the maximum Tsai-Wu failure index is found to be $15 \%$ higher for the inextensible formulation in comparison with the extensible simulation, for the unpressurised riser. However, when the pressure in the riser increases to 200 bar then the percentage difference between RiSim and Flexcom decreases to 2.3\% as shown in table 8 Although the percentage difference is attributed to higher oscillations observed with the in-extensible formulation as explained in section 3.2.2 The TsaiWu failure indices are therefore similar and the formulations don't have a large effect on the composite failure index in strength conditions. The percentage difference is noticed to decrease with higher internal pressure as shown in table 8 Based on the presented results a reliability analysis or dynamic fatigue simulation is expected to predict marginally higher probabilities of failure if the in-extensible method is used compared to the extensible model. However, these differences are currently captured within the recommended high safety factors that are used in the composite riser design. The finite element analysis running time is 6.8 minutes to simulate 74.5 seconds of real time on an Intel(R) Core(TM) i7-6700 CPU @ 3.40GHz with 31 GB RAM computer.

Table 8: Tsai-Wu failure index

\begin{tabular}{lrr}
\hline Global model & \multicolumn{2}{c}{ Tsai-Wu failure index } \\
& Unpressurised & Pressurised \\
\hline RiSim & 0.014 & 0.101 \\
Flexcom & 0.016 & 0.103 \\
\hline
\end{tabular}




\section{Conclusion}

As the industry increases the use of composite risers there is a need to review the current techniques used in design. This paper compares models of the global riser and pipe response to determine their appropriateness for composite riser applications. The literature is divided into two main methods for global riser behaviour: extensible and in-extensible beam formulations. In this paper RiSim is developed based on an extensible formulation and compared to an in-extensible FE riser commercial package Flexcom. Both methods predict similar maximum tensions and curvatures with less than $1 \%$ difference. However, for the environmental loading conditions listed in table 3 and sinusoidal top-side excitation following equation 11 , the riser tension predicted by the in-extensible formulation experiences spurious high frequency oscillations, although these reduce after the first three wave periods. The source of such oscillations is expected to originate from the in-extensibility condition applied to risers that exhibit lower axial stiffness than flexible steel risers, therefore user care is required during the post-processing stage. The extensible finite difference solution doesn't suffer from spurious oscillations and requires minimal user intervention, and is therefore more suitable for efficient automation and digital-twinning applications, especially those involving supervised learning where these oscillations could affect the machine learning. Composite elements are compared where continuum shell, SC8R, and quadratic shell, S8R, elements are found to be the most suitable to model composite pipe cross-sections. The cross-sectional stresses predicted by the pipe FE model coupled with the in-extensible formulation predicts a Tsai-Wu failure index that is marginally higher than predicted with the extensible formulation. These higher stresses do not have a large effect on the strength response of the composite pipe.

\section{Acknowledgements}

This work was supported by EPSRC Centre of Doctoral Training grant no. EP/L015382/1. We thank wood company for providing academic license of their riser analysis software Flexcom which is used to benchmark the results published in this paper. We would also like to thank Lloyds Register Foundation for supporting this research.

\section{References}

[1] F. B. D. A. Carr, Malcolm; Sinclair, Pipeline walking understanding the field layout challenges and analytical solutions developed for the safebuck jip SPE Projects Facilities and Construction 3. doi : safebuckJIP URL https://www . onepetro.org/journal-paper/SPE-120022-PA

[12] A. Reda, I. A. Sultan, I. M. Howard, G. L. Forbes, K. K. McKee, Pipeline walking and anchoring considerations in the presence of riser

motion and inclined seabed International Journal of Pressure Vessels and Piping 162 (2018) 71 - 85. doi:https://doi.org/10.1016/ j.ijpvp.2018.01.003 URL http://www.sciencedirect.com/science/article/pii/S0308016117303824

[3] A. Reda, K. K. McKee, I. M. Howard, I. A. Sultan, When is a subsea anchor required for a short pipeline/scr system? International Journal of Pressure Vessels and Piping 171 (2019) 278 - 298. doi:https://doi.org/10.1016/j.ijpvp.2019.02.009 URL http://www.sciencedirect.com/science/article/pii/S0308016118304204 
[4] Y. M. Tarnopol'skii, V. L. Kulakov, D. D. Mungalov, Composites in offshore technology in the next century, Mechanics of Composite Materials 35 (5) (1999) 365-372. doi:10.1007/BF02329321

[5] O. O. Ochoa, M. M. Salama, Offshore composites: Transition barriers to an enabling technology, Composites Science and Technology 65 (2005) 2588-2596. doi:10.1016/j.compscitech.2005.05.019

[6] A. I. Beyle, C. G. Gustafson, V. L. Kulakov, Y. M. Tarnopol'skii, Composite risers for deep-water offshore technology: Problems and prospects. 1. Metal-composite riser, Mechanics of Composite Materials 33 (5) (1997) 403-414. doi : 10.1007/BF02256894

[7] A. M. Reda, G. L. Forbes, F. Al-Mahmoud, I. M. Howard, K. K. McKee, I. A. Sultan, Compression limit state of hvac submarine cables. Applied Ocean Research 56 (2016) 12 - 34. doi:https://doi.org/10.1016/j.apor.2016.01.002 URL http://www.sciencedirect.com/science/article/pii/S0141118716000031

[8] D.-C. Pham, N. Sridhar, X. Qian, A. J. Sobey, M. Achintha, A. Shenoi, A review on design, manufacture and mechanics of composite risers, Ocean Engineering 112 (2016) 82-96. doi:10.1016/j.oceaneng. 2015.12.004

口 [9] M. Patel, F. Seyed, Review of flexible riser modelling and analysis techniques, Engineering Structures 17 (4) (1995) 293 - 304. doi: http: //dx.doi.org/10.1016/0141-0296(95)00027-5

[10] S. Timoshenko, D. H. Young, W. Weaver, Vibration Problems in Engineering, Vol. 208, 1990. doi:10.1038/208964b0

[11] R. P. Nordgren, On computation of the motion of elastic rods, Journal of Applied Mechanics 41. doi:10.1115/1.3423387

[12] D. L. Garrett, Dynamic analysis of slender rods, Journal of Energy Resources Technology 104. doi:10.1115/1.3230419

[13] T. Huang, S. Chucheepsakul, Large displacement analysis of a marine riser, Journal of Energy Resources Technology, Transactions of the ASME 107 (1) (1985) 54-59.

[14] M. M. Bernitsas, J. E. Kokarakis, Importance of nonlinearities in static riser analysis, Applied Ocean Research 10 (1) (1988) 2 - 9. doi: https://doi.org/10.1016/S0141-1187(88)80019-1

[15] J. F. McNamara, P. J. OBrien, S. G. Gilroy, Nonlinear Analysis of Flexible Risers Using Hybrid Finite Elements, Journal of Offshore Mechanics and Arctic Engineering 110 (3) (1988) 197-204.arXiv:https://asmedigitalcollection.asme.org/offshoremechanics/ article-pdf/110/3/197/4981764/197\_1.pdf doi:10.1115/1.3257051

[16] P. J. O’Brien, J. F. McNamara, Analysis of Flexible Riser Systems Subject to Three-Dimensional Seastate Loading, Int. Conf. on Behaviour of Offshore Structures (BOSS '88) (1988) 1373-1388.

[17] P. O'Brien, J. McNamara, Significant characteristics of three-dimensional flexible riser analysis, Engineering Structures 11 (4) (1989) 223 233. doi:https://doi.org/10.1016/0141-0296(89)90041-2

[18] B. D. Edmans, D. C. Pham, Z.-Q. Zhang, T. F. Guo, N. Sridhar, G. Stewart, An Effective Multiscale Methodology for the Analysis of Marine Flexible Risers. Journal of Marine Science and Engineering 7 (10) (2019) 340. doi:10.3390/jmse7100340 URL https://www.mdpi.com/2077-1312/7/10/340

[19] I. Fried, Large deformation static and dynamic finite element analysis of extensible cables, Computers and Structures 15 (3) (1982) 315 319. doi:http://dx.doi.org/10.1016/0045-7949(82)90022-0

[20] F. A. Leckie, G. Lindberg, The effect of lumped parameters on beam frequencies, Aeronautical Quarterly 14 (3) (1963) 224240. doi: 10.1017/S0001925900002791

[21] P. G. Bergan, E. Mollestad, N. Sandsmark, Nonlinear static and dynamic response analysis for floating offshore structures, Engineering Computations 2 (1) (1985) 13-20. arXiv:http://dx.doi.org/10.1108/eb023596 doi:10.1108/eb023596

[22] M. Casarella, M. Parsons, Cable systems under hydrodynamic loading., Marine Technology Society Journal 4 (4) (1970) $27-44$.

[23] M. J. Choc, Young-Il; Casarella, Configuration of a towline attached to a vehicle moving in a circular path, Journal of Hydronautics 6. doi:10.2514/3.62894 
[24] C. Ablow, S. Schechter, Numerical simulation of undersea cable dynamics, Ocean Engineering 10 (6) (1983) 443 - 457. doi:http: //dx.doi.org/10.1016/0029-8018(83)90046-X

[25] F. S. Hover, M. A. Grosenbaugh, M. S. Triantafyllou, Calculation of dynamic motions and tensions in towed underwater cables, IEEE Journal of Oceanic Engineering 19 (3) (1994) 449-457.

[26] M. S. Triantafyllou, The dynamics of taut inclined cables, The Quarterly Journal of Mechanics and Applied Mathematics 37 (3) (1984)

421-440. arXiv:/oup/backfile/content_public/journal/qjmam/37/3/10.1093/qjmam/37.3.421/2/37-3-421.pdf doi: $10.1093 / q j m a m / 37.3 .421$

[27] I. K. Chatjigeorgiou, A finite differences formulation for the linear and nonlinear dynamics of $2 \mathrm{~d}$ catenary risers, Ocean Engineering 35 (7) (2008) 616 - 636. doi:https://doi.org/10.1016/j.oceaneng.2008.01.006

[28] S. A. Chatjigeorgiou, Ioannis K.and Mavrakos, Cable Dynamics for Marine Applications, Springer International Publishing, Cham, 2016, pp. 875-906. doi:10.1007/978-3-319-16649-0_38

[29] M. J. D. Powell, An efficient method for finding the minimum of a function of several variables without calculating derivatives, The Computer Journal 7 (2) (1964) 155-162. arXiv:http://oup.prod.sis.lan/comjnl/article-pdf/7/2/155/959784/070155.pdf doi:10. 1093/comjnl/7.2.155

[30] K. V. P. K. Vajravelu, Keller-Box method and Its Application Berlin, Boston: De Gruyter, 2014. URL https://www.degruyter.com/view/product/180086

[31] S. D. C. Rakshit, T.; Atluri, Viv of a composite riser at moderate reynolds number using cfd, Journal of Offshore Mechanics and Arctic Engineering 130. doi:10.1115/1.2783849

[32] W. (2018), Flexcom Theory Manual, Wood.

[33] C. M. Larsen, Flexible riser analysis comparison of results from computer programs Marine Structures 5 (2) (1992) 103-119. doi:https: //doi.org/10.1016/0951-8339(92)90024-J URL http://www.sciencedirect.com/science/article/pii/095183399290024J

[34] J. Burgess, M. Triantafyllou, The elastic frequencies of cables, Journal of Sound and Vibration 120 (1) (1988) 153 - 165. doi:https: //doi.org/10.1016/0022-460X(88)90340-9

[35] J.N.Reddy, Mechanics of Laminated Composite Plates and Shells, 2nd Edition, 2003. doi:https://doi.org/10.1201/b12409

[36] A. J. Sadowski, J. M. Rotter, Solid or shell finite elements to model thick cylindrical tubes and shells under global bending, International Journal of Mechanical Sciences 74 (2013) 143 - 153. doi:https://doi.org/10.1016/j.ijmecsci.2013.05.008

[37] C. Wang, K. Shankar, E. V. Morozov, Tailored local design of deep sea frp composite risers, Advanced Composite Materials 24 (4) (2015) 375-397. arXiv:https://doi.org/10.1080/09243046.2014.898438 doi:10.1080/09243046.2014.898438

[38] X. S. Sun, Y. Chen, V. B. C. Tan, R. K. Jaiman, T. E. Tay, Homogenization and Stress Analysis of Multilayered Composite Offshore Production Risers, Journal of Applied Mechanics 81 (3) (2013) 031003. doi :10.1115/1.4024695 